\title{
Time-frequency reassignment: from principles to algorithms
}

\section{P. Flandrin}

Ecole Normale Superieure de Lyon

F. Auger

Universite de Nantes

E. Chassande-Mottin

Observatoire de la Cote d'Azur

\subsection{Introduction}

Time-frequency analysis (TF) is a field that has experienced a number of qualitative and quantitative changes during the last two decades. Whereas most of classical signal processing studies of the 1970s were aimed at stationary signals and processes, many efforts were devoted to less idealized situations during the 1980s, and the idea of TF progressively emerged as a new paradigm for nonstationarity. It is now well recognized that many signal processing problems can be advantageously phrased in a TF language, and the issue may no longer be designing brand new methods from scratch, but instead in adequately using some of the many tools that we have at our disposal, or in improving them for specific tasks. In some sense, the purpose of this chapter has to be understood from this second generation perspective, because what is discussed here essentially builds on the methods that have already been extensively 
studied and used. New advances nevertheless are to be provided, thanks to fresh interpretations that have been made possible by recent developments in TF analysis.

This chapter is devoted to reassignment, a technique that was first introduced in the 1970s in a somehow restricted framework, with a scope that has been substantially enlarged, thanks to the new developments that modern TF analysis has experienced. If the concerns are what reassignment, is and what it is good for, the explanation is at least twofold. First, reassignment can be viewed as a postprocessing technique aimed at improving readability of TF distributions (exploratory signal analysis). Second, reassignment can be used as a way of coding useful TF information, so as to be part of decision schemes (signal processing). We concentrate mainly on the first aspect, referring the interested reader to more comprehensive treatments concerning the second one [11].

More precisely, the chapter is organized as follows. Section 5.2 first motivates the usefulness of reassignment by stressing how it permits to overcome the localization and interference trade-off that is usually observed in classical TF analysis. The reassignment principle then is to be detailed in the simplest case of the spectrogram, and some examples illustrate different facets of the technique. Section 5.3 focuses on reassignment in action: starting from the spectrogram case, efficient algorithmic issues are discussed, as well as extensions to more general situations, including time-scale distributions such as the scalogram (squared wavelet transform). Finally, Section 5.4 points out a number of real-world situations where reassignment may be of effective usefulness, both in exploratory data analysis and in signal manipulation.

The point of view adopted in this chapter is mostly practical, with as little theory as needed (for more fundamental aspects, the interested reader is referred to [2] or [11]). Throughout the text, and for a sake of illustration, an extensive use is made of MATLAB routines that are part of a freeware toolbox [3], downloadable from:

\section{http://iut-saint-nazaire.univ-nantes.fr/ auger/tftb.html}

The specific procedures used are available from the universal resource locator (URL).

http://iut-saint-nazaire.univ-nantes.fr/ auger/publis/CRC.html

and they must be explicitly considered as part of the chapter, because they allow the readers not only to reproduce the figures used as illustrations but also to make their own variations on their production, so as to become more familiar with the proposed tools.

\subsection{Reassigned time-frequency distributions}

\subsubsection{Motivation example}

TF tools are extensively used for exploratory signal analysis. To address some of the problems that conventional TF tools are faced with, let us first consider the example of Figure 5.1, produced by running model1plot. 


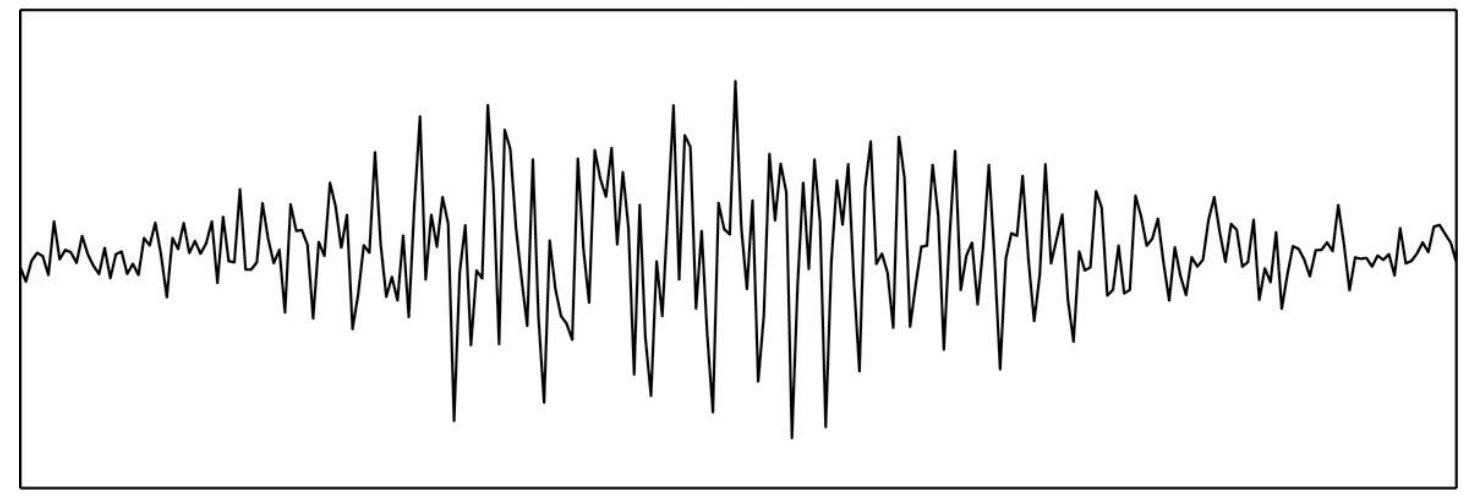

time

spectrum $|X(f)|$

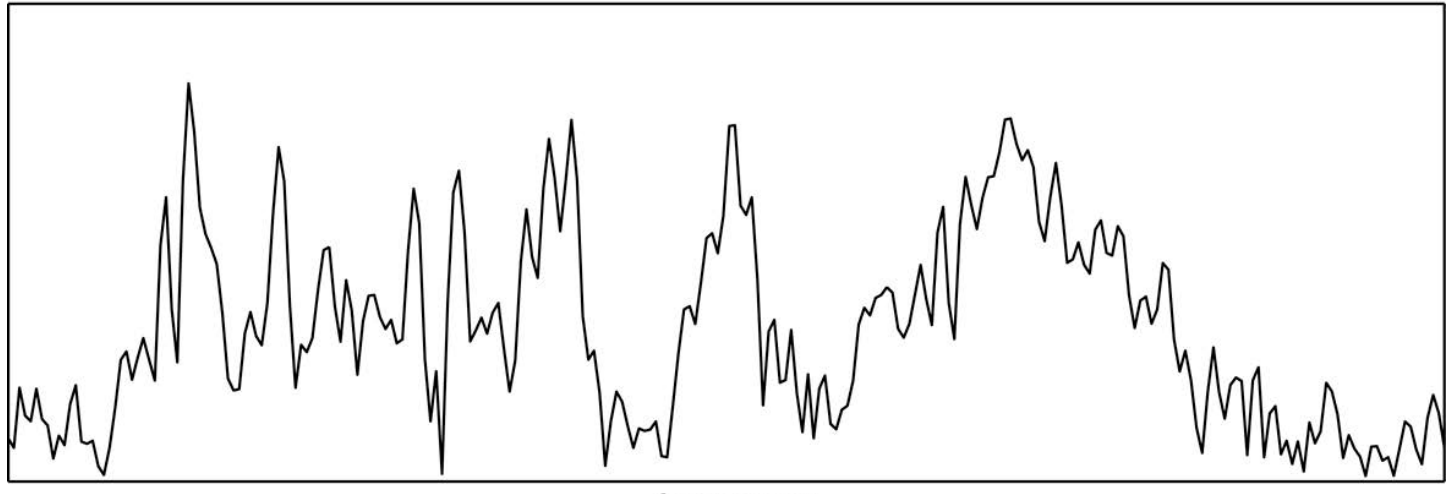

frequency

\section{FIGURE 5.1}

A three-component signal embedded in noise. From a point of view of exploratory data analysis, neither the waveform (top) nor its spectrum (bottom) adequately reveals the actual structure of the analyzed signal - Equation (5.1).

In fact, looking at the waveform or at its spectrum does not allow for a simple interpretation of the inner structure of the signal $\mathbf{x}$, which consists in a linear combination of 3 different amplitude-modulated-frequency-modulated (AM-FM) components embedded in noise with a 20-dB Signal-to-noise ratio (SNR), generated according to:

$$
\begin{aligned}
& \mathrm{N}=256 ; \text { fmins }=0.05 ; \text { fmaxs }=0.2 \\
& {[\mathrm{x}, \mathrm{fxs}, \mathrm{fxl}, \mathrm{dt}]=\operatorname{model} 1(\mathrm{~N}, \mathrm{fmins}, \mathrm{fmaxs}, 0.45,0.25, \mathrm{~N} / 3,0.25,20)}
\end{aligned}
$$

In such a situation, a much clearer insight would be gained by an explicit description of the time-frequency structure of each of the components. The relevance of such a mixed description is supported by Figure 5.2, obtained by running model1TF. Indeed, the simplified model displayed in the top left subplot of Figure 5.2 makes apparent the coexistence of two FM components (one sinusoidal and one linear, of respective instantaneous frequencies fxs and fxl) and one logon (Gaussian wave packet of effective duration dt). The purpose of exploratory TF analysis is therefore to produce a picture as close to this idealized model as possible, given the observed three-component signal, of a priori unknown structure.

Because of its many theoretical properties [16], the Wigner-Ville distribution (WVD) could be thought of as the most appropriate tool, but it appears (top right 

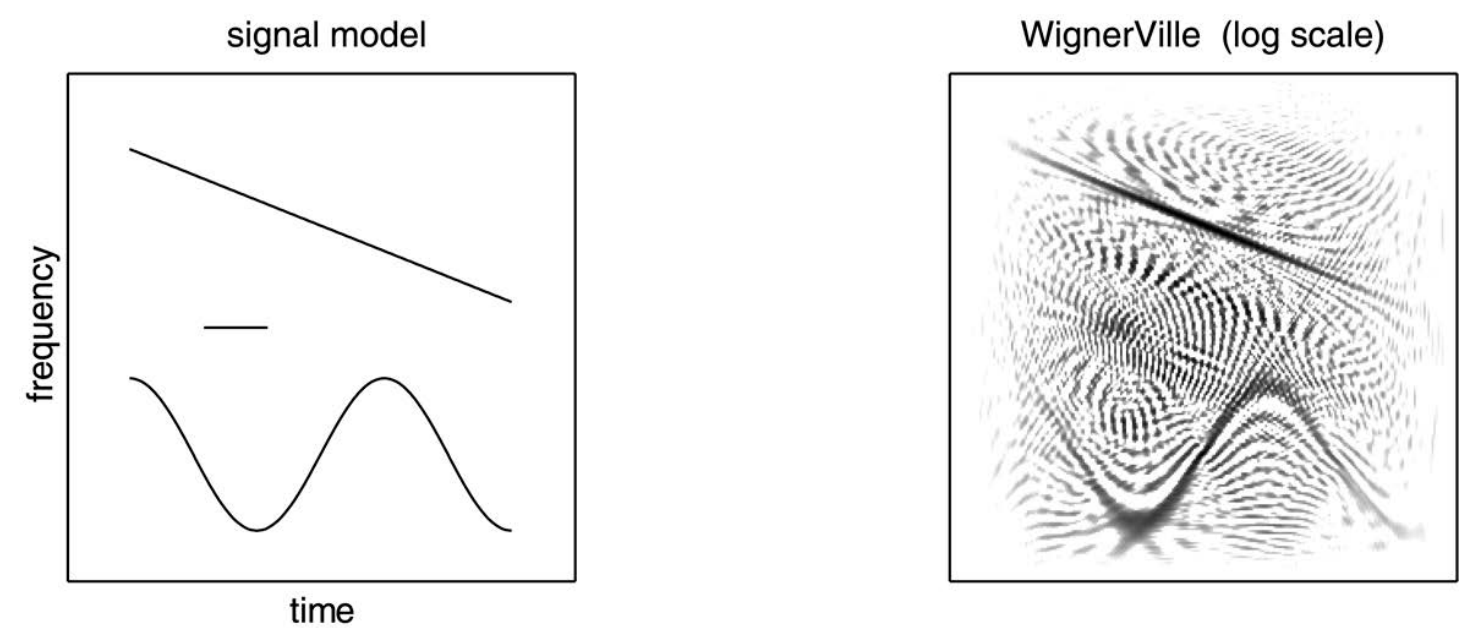

spectrogram (log scale)

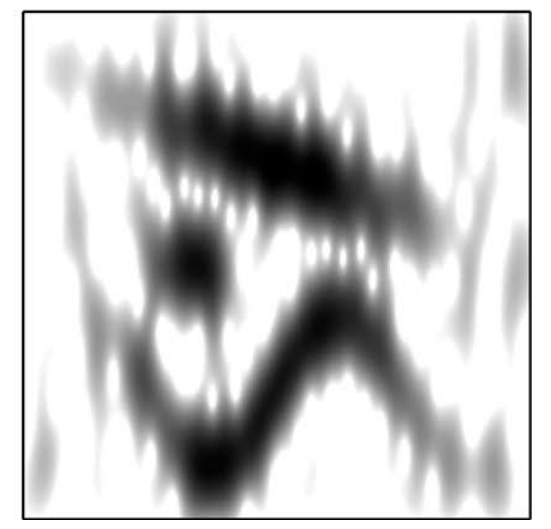

reassigned spectro. (log scale)

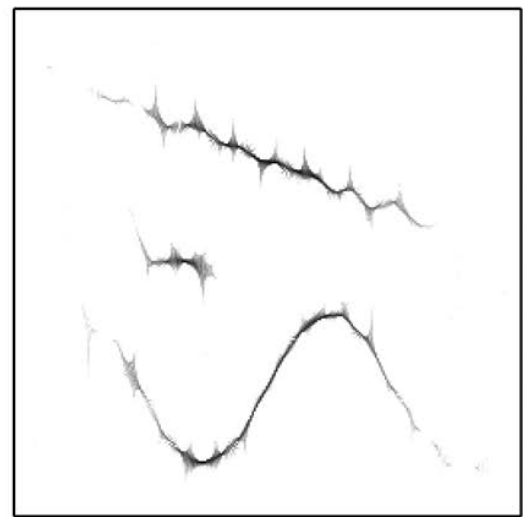

\section{FIGURE 5.2}

A three-component signal embedded in noise. The idealized time-frequency model is compared with three images produced by the Wigner-Ville distribution (WVD), a spectrogram, and a reassigned spectrogram. Whereas the readability of the WVD (top right) is hampered by oscillatory interference terms and the spectrogram (bottom left) suffers from a poor resolution, the reassigned spectrogram (bottom right) provides the user with a time-frequency picture which is almost identical to the idealized model (top left).

of Figure 5.2) that, whereas individual components are rather sharply described, the overall readability is hampered by cross-components interference phenomena [23]. As is well-known, a spectrogram (bottom left of Figure 5.2) contains much less crossterms, but this image cleaning is obtained at the expense of a smearing of the TF signatures of the individual components. Overcoming this trade-off between localization and interference is precisely one of the "raisons d'être" of reassignment, whose application results in a picture (bottom right of Figure 5.2) that is almost identical to the idealized model we started with.

\subsubsection{Reassignment basics}

To understand how the miracle of Figure 5.2 occurs, it is worth going back to basic definitions and properties of classical TF distributions. 


\subsubsection{From spectrograms to Wigner-Ville}

First, a spectrogram $S_{x}^{h}(t, \omega)$ is usually defined as:

$$
S_{x}^{h}(t, \omega):=\left|F_{x}^{h}(t, \omega)\right|^{2}
$$

where $F_{x}^{h}(t, \omega)$ stands for the short-time Fourier transform (STFT):

$$
F_{x}^{h}(t, \omega):=\int_{-\infty}^{+\infty} x(s) h^{*}(s-t) e^{-\imath \omega s} d s \times e^{\imath t \omega / 2}
$$

Those definitions are explicitly dependent on some short-time window $h(t)$, aimed at limiting the evaluation of the Fourier transform (FT) to some specified neighborhood of the current date $t$. The window introduced this way may be thought of as a measurement device, with the consequence that the produced distribution jointly depends on the signal and on the window. In particular, STFTs and spectrograms are faced with a TF resolution trade-off, because the shorter the duration of $h(t)$, the better their resolution in time, but the larger the bandwidth of the spectrum $H(\omega)$ and, henceforth, the poorer their resolution in frequency.

Instead of fixing arbitrarily $h(t)$, one may try to make it depend adaptively on the analyzed signal. Thinking of the STFT as a linear filtering operation, the powerful concept of matched filtering suggests the intuitive choice $h(t)=x_{-}(t):=x(-t)$ (i.e., to take as window the time-reversed version of the analyzed signal). It then follows that:

$$
F_{x}^{x_{-}}(t, \omega)=W_{x}(t / 2, \omega / 2) / 2
$$

where:

$$
W_{x}(t, \omega):=\int_{-\infty}^{+\infty} x(t+s / 2) x^{*}(t-s / 2) e^{-\imath s \omega} d s
$$

is nothing but the WVD [16].

By definition, the WVD does present the advantage of depending only on the signal. Its role is central in TF analysis and it possesses a number of theoretical properties $[12,16]$ among which one can mention its ability to be perfectly localized in the case of linear FM signals. These appealing features have, however, to be paid at some price because, for example, a WVD cannot be positive everywhere, thus forbidding a local density interpretation. Furthermore, in accordance with the elementary identity $(a+b)^{2}=a^{2}+b^{2}+2 a b$, the fully quadratic nature of the WVD is known to create spurious cross terms, characterized by oscillating contributions located midway in between any two interacting components [23].

\subsubsection{Localization vs. interference}

Because interference terms of the WVD are by nature oscillatory, one can think of reducing them by applying some low-pass smoothing, but such a smoothing operation has also the negative effect of spreading out localized signal terms. This trade-off between joint localization and the importance of interference terms is clearly apparent when comparing (as in Figure 5.2) the WVD and a spectrogram, properly interpreted 
as a smoothed WVD. Indeed, instead of using its usual definition, as in Equation (5.2), a spectrogram can be equivalently expressed as [16]:

$$
S_{x}^{h}(t, \omega)=\iint_{-\infty}^{+\infty} W_{x}(s, \xi) W_{h}(s-t, \xi-\omega) \frac{d s d \xi}{2 \pi}
$$

thus making explicit the fact that it results from the smoothing of the signal WVD by the window WVD.

\subsubsection{Reassignment principle}

An important consequence of the smoothing formula in Equation (5.6) is that the value that a spectrogram takes at a given point $(t, \omega)$ of the plane cannot be considered as pointwise, but instead results from the summation of a continuum of WVD contributions within some TF domain defined as the essential TF support of the short-time window. In other words, a whole distribution of values is summarized by a single number, and this number is assigned to the geometric center of the domain over which the distribution is considered.

By reasoning with a mechanical analogy, the situation is as if the total mass of an object were assigned to its geometric center, an arbitrary point that - except in the very specific case of an homogeneous distribution over the domain - has no reason to suit the actual distribution.

A much more meaningful choice is to assign the total mass to the center of gravity of the distribution within the domain, and this is precisely what reassignment does: at each TF point $(t, \omega)$ where a spectrogram value is computed, one also computes the two quantities:

$$
\begin{aligned}
& \hat{t}_{x}(t, \omega):=\frac{1}{S_{x}^{h}(t, \omega)} \iint_{-\infty}^{+\infty} s W_{x}(s, \xi) W_{h}(s-t, \xi-\omega) \frac{d s d \xi}{2 \pi} \\
& \hat{\omega}_{x}(t, \omega):=\frac{1}{S_{x}^{h}(t, \omega)} \iint_{-\infty}^{+\infty} \xi W_{x}(s, \xi) W_{h}(s-t, \xi-\omega) \frac{d s d \xi}{2 \pi}
\end{aligned}
$$

which define the local centroids of the WVD distribution $W_{x}$, as seen through the TF window $W_{h}$ centered in $(t, \omega)$. The spectrogram value is then moved from the point $(t, \omega)$ where it has been computed to this centroid $\left(\hat{t}_{x}(t, \omega), \hat{\omega}_{x}(t, \omega)\right)$, leading to define the reassigned spectrogram as:

$$
\check{S}_{x}^{h}(t, \omega):=\iint_{-\infty}^{+\infty} S_{x}^{h}(s, \xi) \delta\left(t-\hat{t}_{x}(s, \xi), \omega-\hat{\omega}_{x}(s, \xi)\right) \frac{d s d \xi}{2 \pi}
$$

Conceptually, reassignment can be considered a two-step process: (1) a smoothing, whose main purpose is to rub out oscillatory interferences, but whose drawback is to smear localized components; (2) a squeezing, whose effect is to refocus the contributions that survived the smoothing. 


\subsubsection{Some historical comments}

As has been sketched earlier, the presentation of the reassignment principle is a modern way [2] of revisiting a much older idea [25, 27].

The argument used by Kodera, De Villedary and Gendrin in their seminal papers $[25,27]$ was not related to TF smoothing, but to phase. In fact, the centroids as in Equations (5.7) and (5.8), used in reassignment happen to be related to the phase of the STFT, an information that is discarded when considering a spectrogram as a squared STFT. More precisely, denoting by $\varphi(t, \omega)$ the phase of the STFT, as in Equation (5.3), and using the simplified notation $\partial_{u} \varphi=\partial \varphi / \partial u$, local centroids, as in Equations (5.7) and (5.8) can be shown $[25,27]$ to be equivalently given by:

$$
\begin{aligned}
& \hat{t}_{x}(t, \omega)=\frac{t}{2}-\partial_{\omega} \varphi(t, \omega) \\
& \hat{\omega}_{x}(t, \omega)=\frac{\omega}{2}+\partial_{t} \varphi(t, \omega)
\end{aligned}
$$

These quantities can be interpreted as the local instantaneous frequency (IF) and group delay (GD) of the analyzed signal, as filtered within the TF domain defined by the TF window $W_{h}$ centered in $(t, \omega)$. From this interpretation, the rationale for reassignment (initially referred to as a "modified moving window method" [25, 27]) was to favor energy concentrations in the vicinity of local IFs and GDs.

Although introduced in the mid-1970s and applied with success in geophysics, the reassignment technique retained almost no attention of the TF community the next 20 years. Apart from the fact that the methodology has been little publicized per se, the reason is certainly that it had been introduced much before the problems it addressed were extensively studied (during the mid-1980s), and that its applicability remained computationally limited for a while.

Not until the mid-1990s did the idea of reassignment surface again [2]. Algorithmic improvements were proposed and, thanks to the many developments that occurred the field, the scope of the technique was considerably enlarged, far beyond only the spectrogram case. In parallel, other related techniques were developed independently(e.g., the "ridge and skeleton" method $[5,13,19,20]$, the "instantaneous frequency density"[17], the "differential spectral analysis"[18] or the "synchrosqueezing" technique [29]).

\subsubsection{Toy examples}

\subsubsection{Linear chirps}

It is well-known [16] that an idealized linear FM signal has a WVD that is perfectly localized in the TF plane, namely:

$$
x(t)=\exp \left\{\imath\left(\omega_{0} t+\beta t^{2} / 2\right)\right\} \Rightarrow W_{x}(t, \omega)=\delta\left(\omega-\left(\omega_{0}+\beta t\right)\right)
$$

for any modulation rate $\beta$.

Therefore, it may be concluded that reassigned spectrograms automatically inherit of this perfect localization, because the centroid of a line distribution necessarily 
belongs to its (localized) support. It is worth noting that, geometrically, TF lines include pure tones $(\beta=0)$ and impulses $(\beta=\infty)$ as limiting cases. In all cases, any reassigned spectrogram (i.e., whatever the window $h(t)$ it is computed with) is theoretically guaranteed to be perfectly localized.

If we turn to the more realistic situation of a linear chirp, defined as:

$$
x(t)=\exp \left\{-\pi \gamma t^{2}\right\} \times \exp \left\{\imath\left(\omega_{0} t+\beta t^{2} / 2\right)\right\}
$$

explicit calculations can be conducted, but they are not reproduced in this chapter(the interested reader is referred to [27] or [11]). We instead investigate through examples how a reassigned spectrogram jointly depends on the analyzed chirp and on the shorttime window. The proposed numerical experiment consists in generating a $\mathbf{N x}$-points chirp $\mathbf{x}$, sweeping from fmin to fmax, embedded in a $\mathbf{N y}$-points signal $\mathbf{y}$ :

$$
\begin{aligned}
& \mathrm{x}=\operatorname{gchirp}(\mathrm{Nx}, \mathrm{fmin}, \mathrm{fmax}) ; \mathrm{Lx}=\operatorname{length}(\mathrm{x}) \\
& \mathrm{y}=\operatorname{zeros}(\mathrm{Ny}, 1) ; \mathrm{y}(\mathrm{Ny} / 2-\mathrm{Lx} / 2: \mathrm{Ny} / 2+\mathrm{Lx} / 2-1)=\mathrm{x} ;
\end{aligned}
$$

By running:

$$
\operatorname{sprspplot}(\mathrm{y}, \mathrm{dt}, \mathrm{Nb}, \mathrm{Nh}, \mathrm{c}) \text {; }
$$

this signal $\mathbf{y}$ is then analyzed by a spectrogram (with a Gaussian window of length Nh) and its reassigned version, with both TF distributions computed every dt time samples on a frequency grid of $\mathbf{N b}$ bins, and displayed either in color $(c=1)$ or in black and white $(c=0)$. A typical example corresponding to the set of parameters:

$$
\begin{aligned}
& \mathrm{Nx}=16 ; \mathrm{fmin}=0 ; \mathrm{fmax}=0.5 ; \mathrm{Ny}=256 ; \% \text { signal } \\
& \mathrm{Nh}=255 ; \mathrm{dt}=1 ; \mathrm{Nb}=128 ; \mathrm{c}=0 ; \% \mathrm{TFD} \text { and display }
\end{aligned}
$$

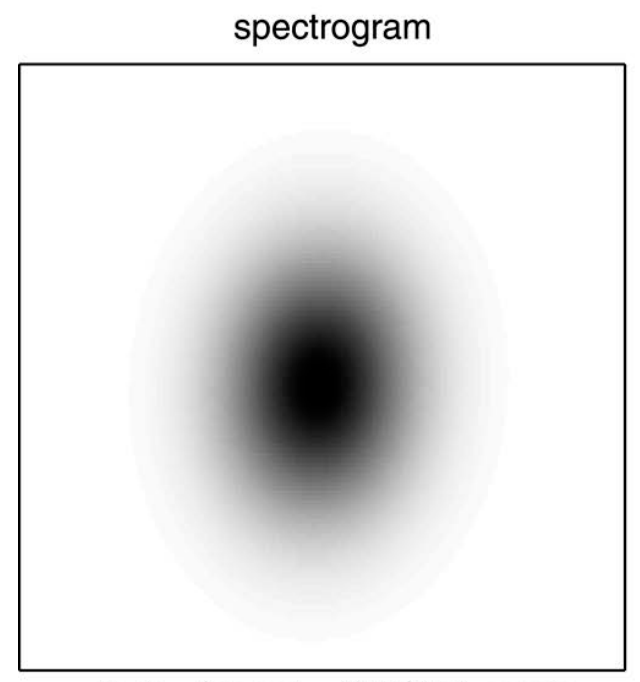

window/signal $=255 / 256$ points

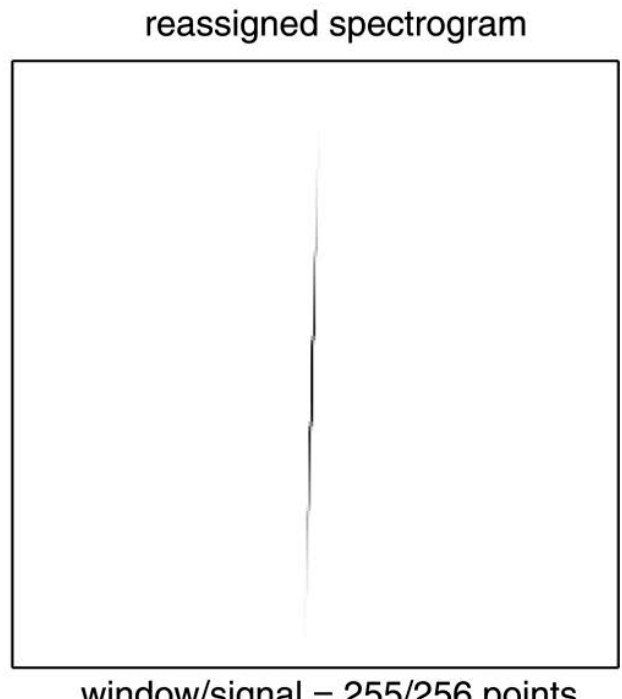

window/signal $=255 / 256$ points

\section{FIGURE 5.3}

Reassigning a short chirp. In the case of a very short transient signal (the effective support of the chirp $x$ considered here is $N x=16$ points, for an observation $y$ of total length $\mathrm{Ny}=\mathbf{2 5 6}$ points) analyzed with a substantially larger window $\mathrm{h}$ ( $\mathrm{Nh}=255$ points), the TF localization is dramatically increased by reassignment. 


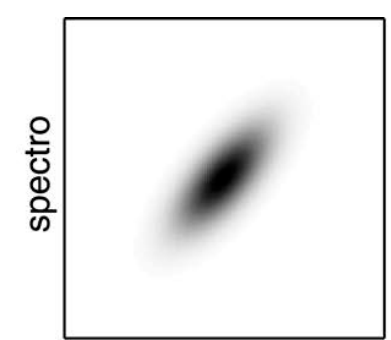

window $=21$

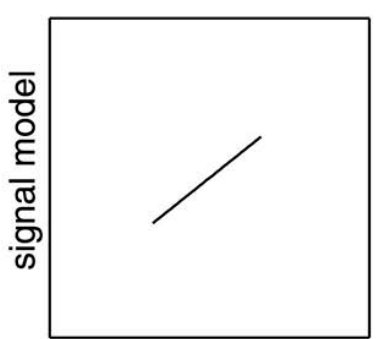

128 points

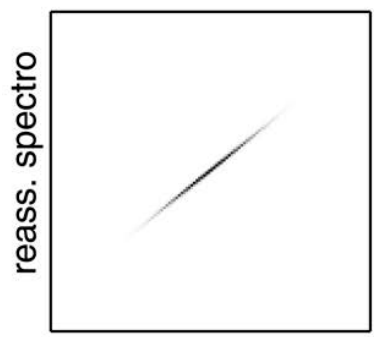

window $=21$

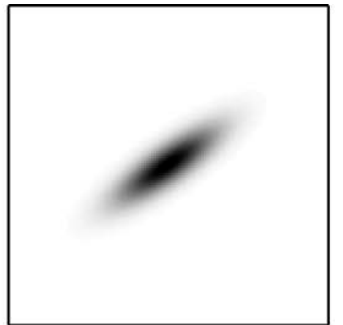

63

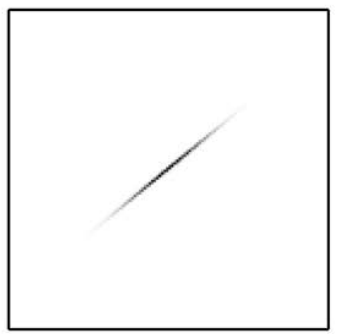

63

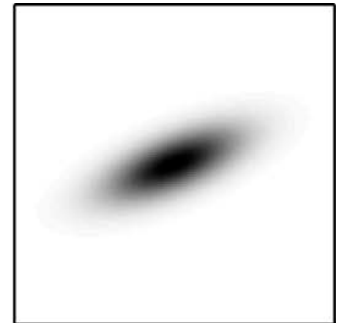

127 points
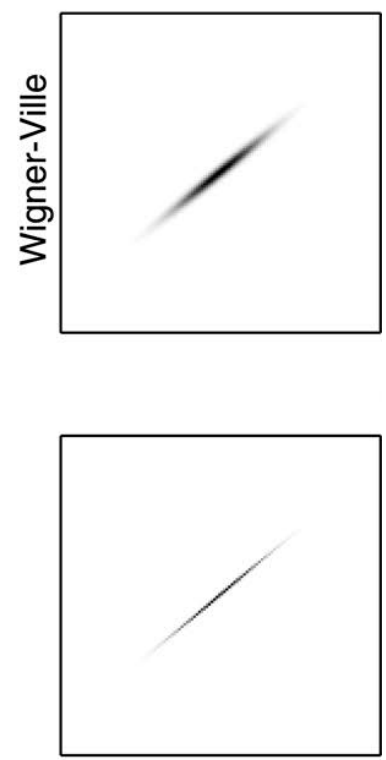

127 points

\section{FIGURE 5.4}

Chirp rate vs. analysis window. When analyzing a chirp signal of fixed chirp rate, the result given by conventional spectrograms (top row) heavily depends on the length of the short-time window. This interdependence between signal and window characteristics is dramatically reduced when replacing spectrograms by their reassigned counterparts (bottom row), leading to sharply localized distributions approaching the idealized model sketched in the left subplot of the middle row. For a sake of comparison, the WVD is displayed in the right subplot of the middle row.

is given in Figure 5.3. It clearly evidences the drastic squeezing effect of reassignment in a situation of a very transient chirp $(\mathbf{N x}=\mathbf{1 6})$ that cannot be considered as quasimonochromatic with respect to the length $(\mathbf{N h}=\mathbf{2 5 5})$ of the analyzing window. Variations on this example are left to the reader.

A more comprehensive comparison is given in Figure 5.4, obtained by running:

$$
\operatorname{varwindow}(\mathrm{Nx}, \mathrm{Nh} 1, \mathrm{Nh} 2, \mathrm{Nh} 3, \mathrm{Nb}, \mathrm{c}) \text {; }
$$

with the specific values:

$$
\begin{array}{lrl}
\mathrm{Nx}=128 ; & & \frac{\circ}{\circ} \text { signal } \\
\mathrm{Nh} 1=21 ; & \text { Nh2 }=63 ; \mathrm{Nh} 3=127 ; & \circ \text { windows } \\
\mathrm{Nb}=128 ; \quad \mathrm{C}=0 ; & \frac{\%}{0} \text { TFD and display }
\end{array}
$$

Whereas, in such a case, conventional spectrograms happen to be poorly localized and to depend heavily on the length of the chosen short-time window, their reassigned 
counterparts prove to always provide fairly similar, correctly localized results (the skeletonized model and the WVD are also given for comparison).

\subsubsection{Nonlinear FM signals}

It has been argued in Section 5.2.3.1 that the reassignment process ends up with a perfect localization in the case of linear FM signals. Conceptually, a similar benefit is expected to be obtained as long as the analyzed signal locally behaves as a linear FM, locality referring to the TF support of the smoothing window. This interdependence between the window length and the local modulation rate can be dynamically observed by running:

$$
\text { quasilin( } \mathrm{Nx}, \mathrm{Nh}, \mathrm{Nb}, \mathrm{Nhp}, \mathrm{c}) \text {; }
$$

where Nx stands for the length of a Nhp half-periods sinusoidal FM signal, and Nh stands for the length of the short-time window used in computing its reassigned spectrogram over $\mathbf{N b}$ frequency bins.

Setting for instance the parameter values to:

$$
\begin{array}{lll}
\mathrm{Nx}=256 ; & \mathrm{Nhp}=21 ; & \frac{\circ}{\circ} \text { signal } \\
\mathrm{Nh}=19 ; & & \frac{\circ}{\circ} \text { window } \\
\mathrm{Nb}=128 ; & \mathrm{C}=0 ; & \% \text { TFD and display }
\end{array}
$$

leads, at time $\mathbf{t}=\mathbf{c e i l}(\mathbf{1 5} * \mathbf{N x} / \mathbf{2 1})$, to the snapshot given in Figure 5.5. What is evidenced by this image is that (slight) departures from localization are observed only in those regions where the local IF trajectory of the model cannot be considered as quasilinear within the TF smoothing window centered at the point of interest.

\subsubsection{Localization vs. resolution}

Whereas reassigning a spectrogram has been shown to end up with a sharply localized distribution, care has to be taken in interpreting this behavior in terms of resolution (i.e., in the ability of separating closely spaced components). In fact, when more than one component is seen within the TF smoothing window, a beating effect occurs and results in interference fringes, thus preventing the consideration of reassignment as some superresolution process. This can be illustrated by running:

$$
\operatorname{resol}(\mathrm{Nx}, \mathrm{Nh}, \mathrm{Nb}, \mathrm{c}) \text {; }
$$

in which a series of crossing linear FM signals of length $\mathbf{N x}$ and of various chirp rates is analyzed over $\mathbf{N b}$ frequency bins, with the same short-time window of length $\mathbf{N h}$. Choosing for instance:

$$
\begin{array}{ll}
\mathrm{Nx}=128 ; & \frac{\%}{\circ} \text { signal } \\
\mathrm{Nh}=31 ; & \frac{\%}{0} \text { window } \\
\mathrm{Nb}=128 ; \quad \mathrm{C}=0 ; & \% \text { TFD and display }
\end{array}
$$

leads to the result displayed in Figure 5.6. Interference effects (emphasized by using a logarithmic scale for the amplitude) are clearly apparent in those regions where the two chirps are simultaneously present within the TF smoothing window. 

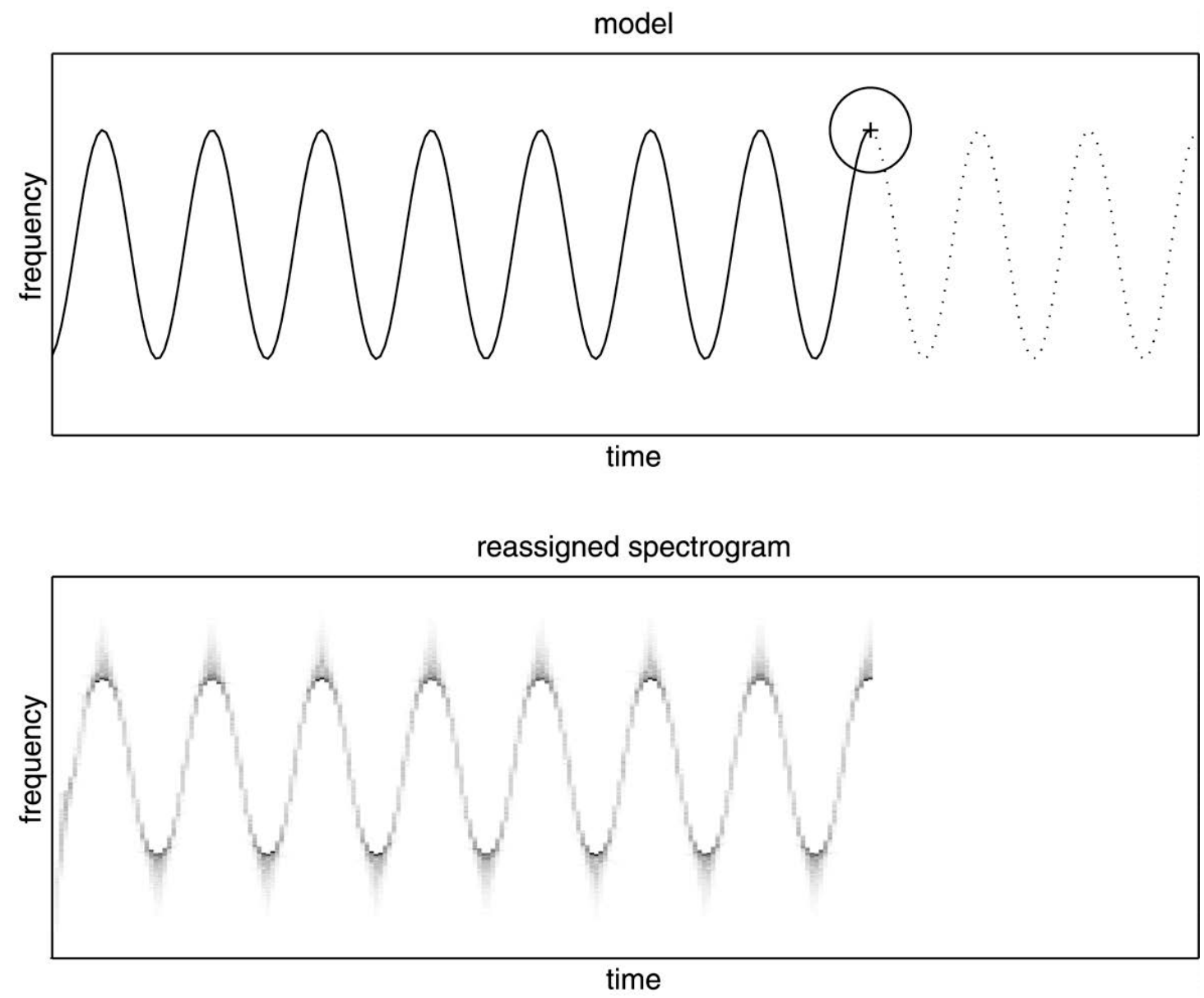

FIGURE 5.5

Local quasilinearity and localization. Due to the local action of reassignment, the perfect localization property that holds for linear FM signals carries over to locally quasilinear situations. In this example of a sinusoidal FM signal, localization is (slightly) degraded at those points where the instantaneous frequency trajectory cannot be consider as quasilinear within the TF smoothing window (represented by the ellipse).

A similar effect can be dynamically observed by visualizing the animation model1 TFmovie.gif, in which a signal of the model1 type is considered, with a logon whose center follows a TF trajectory crossing (back and forth) the linear FM component.

\subsection{Reassignment in action}

In Section 5.2.3, we show that reassignment yields significantly improved TF representations. The present section, devoted to computer algorithms, shows that the computational cost paid for this improvement is not as heavy, and does not prevent the use of this method in practical cases. 

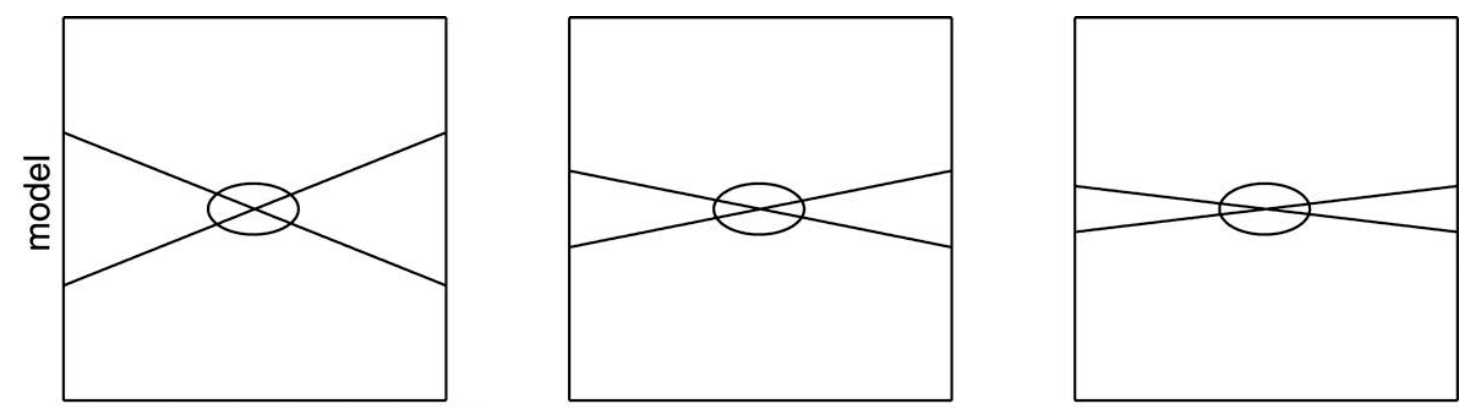

window/signal $=31 / 128$ points
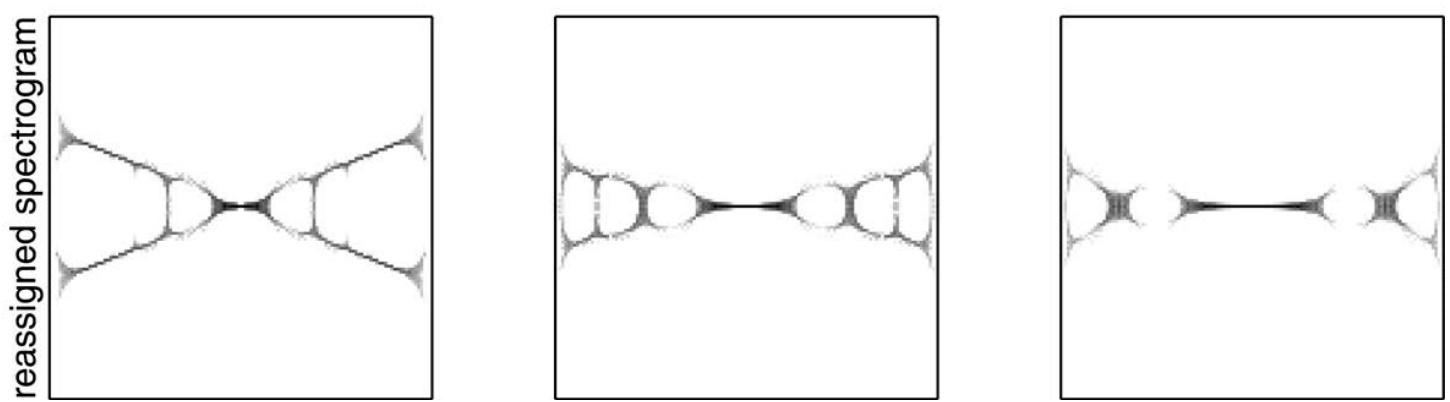

\section{FIGURE 5.6}

Localization and resolution. When more than one component is seen within the TF smoothing window (represented by the ellipse), a beating effect occurs and results in interference fringes. This behavior is illustrated here by two crossing linear FM signals of various chirp rates.

\subsubsection{Algorithms for spectrograms}

\subsubsection{Reassignment operators deduced from phase differences}

As mentioned in Section 5.2.2.4, the reassignment operators $\hat{t}_{x}(t, \omega)$ and $\hat{\omega}_{x}(t, \omega)$ have been initially deduced from the phase of the STFT. These expressions have been used in the first implementations of the reassignment principle [26]. For a signal sampled with a sampling period $T_{s}$ and a frequency axis obtained with a fast Fourier transform (FFT) of length $N$, the partial derivatives of the phase were replaced by first-order differences:

$$
\begin{aligned}
\partial_{t} \varphi[n, m] & \approx \frac{\varphi[n+1, m]-\varphi[n-1, m]}{2 T_{s}} \\
& \approx \frac{1}{2 T_{s}} \arg \left(F_{x}^{h}[n+1, m] F_{x}^{h}[n-1, m]^{*}\right) \\
\partial_{\omega} \varphi[n, m] & \approx \frac{\varphi[n, m+1]-\varphi[n, m-1]}{\left(4 \pi / N T_{s}\right)} \\
& \approx \frac{N T_{s}}{4 \pi} \arg \left(F_{x}^{h}[n, m+1] F_{x}^{h}[n, m-1]^{*}\right)
\end{aligned}
$$

where $F_{x}^{h}[n, m]$ and $\varphi[n, m]$ are, respectively, the STFT value and its phase at time $t=n T_{s}$ and angular frequency $\omega=\frac{2 \pi m}{N T_{s}}$. However, this approach suffers from 
the phase unwrapping problem [33], and yields only approximated values of the reassignment operators.

\subsubsection{More reliable algorithm}

Expressions in Equations (5.15) and (5.17) suggest relating the partial derivatives of the phase to the partial derivatives of the STFT. Defining $G_{x}^{h}(t, \omega)=F_{x}^{h}(t, \omega) e^{-i \omega t / 2}$ $=\left|F_{x}^{h}(t, \omega)\right| e^{i(\varphi-\omega t / 2)}$ leads to:

$$
\begin{aligned}
\partial_{t} G_{x}^{h}(t, \omega) & =-\int_{-\infty}^{+\infty} x(s) \frac{d h^{*}}{d t}(s-t) e^{-\imath \omega s} d s=-G_{x}^{d h}(t, \omega) \\
& =\partial_{t}\left|F_{x}^{h}(t, \omega)\right| e^{i(\varphi-\omega t / 2)}+i\left(\partial_{t} \varphi-\omega / 2\right) G_{x}^{h}(t, \omega) \\
\Longrightarrow \quad \hat{\omega}_{x}(t, \omega) & =\omega-\mathcal{I} m\left\{\frac{G_{x}^{d h}(t, \omega)}{G_{x}^{h}(t, \omega)}\right\}
\end{aligned}
$$

where $\operatorname{Im}\{Z\}$ stands for the imaginary part of the complex number $Z$, and $d h$ is the derivative of the analysis window $d h(t)=\frac{d h}{d t}(t)$. The partial derivative of the phase with respect to the angular frequency leads to a similar expression for $\hat{t}_{x}(t, \omega)$ :

$$
\begin{aligned}
\partial_{\omega} G_{x}^{h}(t, \omega) & =-i\left(G_{x}^{t h}(t, \omega)+t G_{x}^{h}(t, \omega)\right) \\
& =\partial_{\omega}\left|F_{x}^{h}(t, \omega)\right| e^{i(\varphi-\omega t / 2)}+i\left(\partial_{\omega} \varphi-t / 2\right) G_{x}^{h}(t, \omega) \\
\Longrightarrow \quad \hat{t}_{x}(t, \omega) & =t+\mathcal{R} e\left\{\frac{G_{x}^{t h}(t, \omega)}{G_{x}^{h}(t, \omega)}\right\}
\end{aligned}
$$

with $t h(t)=t h(t)$.

Approximated phase differentiations can then be avoided by computing two additional STFTs. These STFTs use the same signal values as $F_{x}^{h}(t, \omega)$, and only differ by their analysis windows. As a consequence, these new expressions do not imply a drastic increase of computational complexity, and can be computed in parallel. It should also be noted that if $h(t)=e^{-\alpha t^{2}}$, a choice that corresponds to the socalled Gabor spectrogram, then $\frac{d h}{d t}(t)=-2 \alpha t h(t)$ and $G_{x}^{d h}(t, \omega)=-2 \alpha G_{x}^{t h}(t, \omega)$. Only one supplementary STFT is required, which makes the reassignment process still faster. This particular case is implemented in the function tfrrgab of the TF toolbox [3].

For sampled signals and discrete Fourier transforms(DFTs) of length $N$, discrete time and discrete frequency versions of the reassignment operators are defined as:

$$
\begin{aligned}
& \hat{n}_{x}[n, m]:=\frac{1}{T_{s}} \hat{t}_{x}\left(n T_{s}, \frac{2 \pi m}{N T_{s}}\right)=n+\mathcal{R} e\left\{\frac{G_{x}^{T_{s}^{-1} t h}[n, m]}{G_{x}^{h}[n, m]}\right\} \\
& \hat{m}_{x}[n, m]:=\frac{N T_{s}}{2 \pi} \hat{\omega}_{x}\left(n T_{s}, \frac{2 \pi m}{N T_{s}}\right)=m-\frac{N}{2 \pi} \mathcal{I} m\left\{\frac{G_{x}^{T_{s} d h}[n, m]}{G_{x}^{h}[n, m]}\right\}
\end{aligned}
$$

with $T_{s} d h[n]=T_{s} \frac{d h}{d t}\left(n T_{s}\right)$ and $T_{s}^{-1} t h[n]=n h\left(n T_{s}\right)$. 
The computation of the reassigned spectrogram of a signal $\mathbf{x}$ at the time instants given in a row vector $\mathbf{t}$, as done in the function tfrrsp of the TF toolbox [3], can then be achieved in two steps.

\subsection{Step one. The first step computes $G_{x}^{h}[n, m](\mathrm{tfr}), G_{x}^{T_{s}^{-1} t h}[n, m](\mathrm{tf} 2)$ and $G_{x}^{T_{s} d h}[n, m](\mathrm{tf3})$ :}

$[$ xrow, $x$ col $]=\operatorname{size}(x) ;[$ trow, tcol $]=\operatorname{size}(t) ;$

[hrow, hcol] =size (h); Lh= (hrow-1) /2;

\% create and initialize three arrays to zero

$\%$ these arrays have as many rows as frequency bins,

\% and as many columns as time intants

tfr $=\operatorname{zeros}(\mathrm{N}, \mathrm{tcol}) ; \operatorname{tf} 2=\operatorname{zeros}(\mathrm{N}, \mathrm{tcol}) ; \operatorname{tf} 3=\operatorname{zeros}(\mathrm{N}, \mathrm{tcol})$;

$\div$ compute th and dh

Th=h. * [-Lh:Lh]'; Dh=dwindow $(\mathrm{h})$;

\% compute the three STFTs

for $i \mathrm{COl}=1: \mathrm{tcol}$,

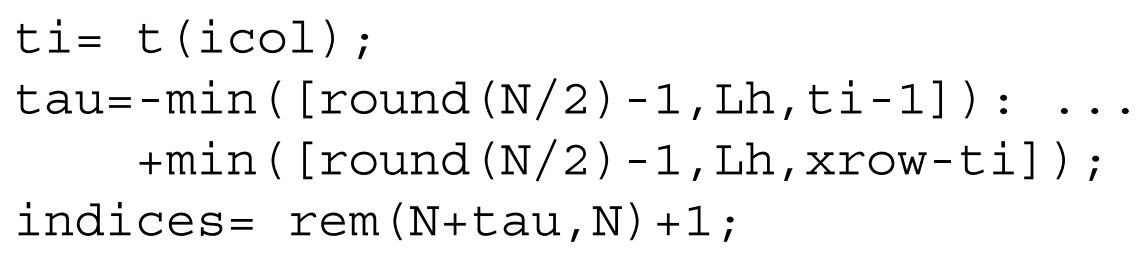

\% normalization to preserve energy norm_h=norm $(\mathrm{h}(\mathrm{Lh}+1+\mathrm{tau}))$;

$\operatorname{tfr}($ indices,$i c o l)=x($ ti+tau $) .{ }^{*} \operatorname{conj}(h(L h+1+t a u)) /$ norm_h; tf $2($ indices, $i c o l)=x($ ti+tau $) .{ }^{*} \operatorname{conj}(T h(L h+1+$ tau $)) /$ norm_h; tf3 $($ indices, $i c o l)=x($ ti+tau $) .{ }^{*} \operatorname{conj}(\mathrm{Dh}(\mathrm{Lh}+1+\mathrm{tau})) /$ norm_h; end ;

\% final call to the FFT function $t f r=f f t(t f r) ; t f 2=f f t(t f 2) ; t f 3=f f t(t f 3)$;

avoidw=find $\left(t f r^{\sim}=0\right) ; \operatorname{tf2}(\operatorname{avoidw})=\operatorname{round}(\operatorname{real}(t f 2(\operatorname{avoidw})$. /tfr(avoidw)/Dt));

tf3 (avoidw) =round (imag $(N * t f 3($ avoidw) .

$/ \operatorname{tfr}(\operatorname{avoidw}) /(2.0 * \mathrm{pi})))$;

$\operatorname{tfr}=\operatorname{abs}(\operatorname{tfr}) \cdot{ }^{\wedge} 2$;

The expression of indices allows the values of the time lag $\tau=\ldots,-2,-1,0,1$, $2, \ldots$ to be located at the indices $\ldots, N-1, N, 1,2,3, \ldots$ as required by the FFT function. In addition, when $F_{x}^{h}[n, m]$ is zero valued, $S_{x}^{h}[n, m]$ is of course also zero valued. Reassignment operators can neither be computed nor be used when $S_{x}^{h}[n, m]$ is zero. This explains the use of the variable avoidw. 


\subsection{Step two. The second step iteratively creates the reassigned} spectrogram by adding $S_{x}^{h}[n, m]$, the value of the spectrogram at the point $\left(n T_{s}, \frac{2 \pi m}{N T_{s}}\right)$ to the value of $\check{S}_{x}^{h}[\hat{n}, \hat{m}]:$

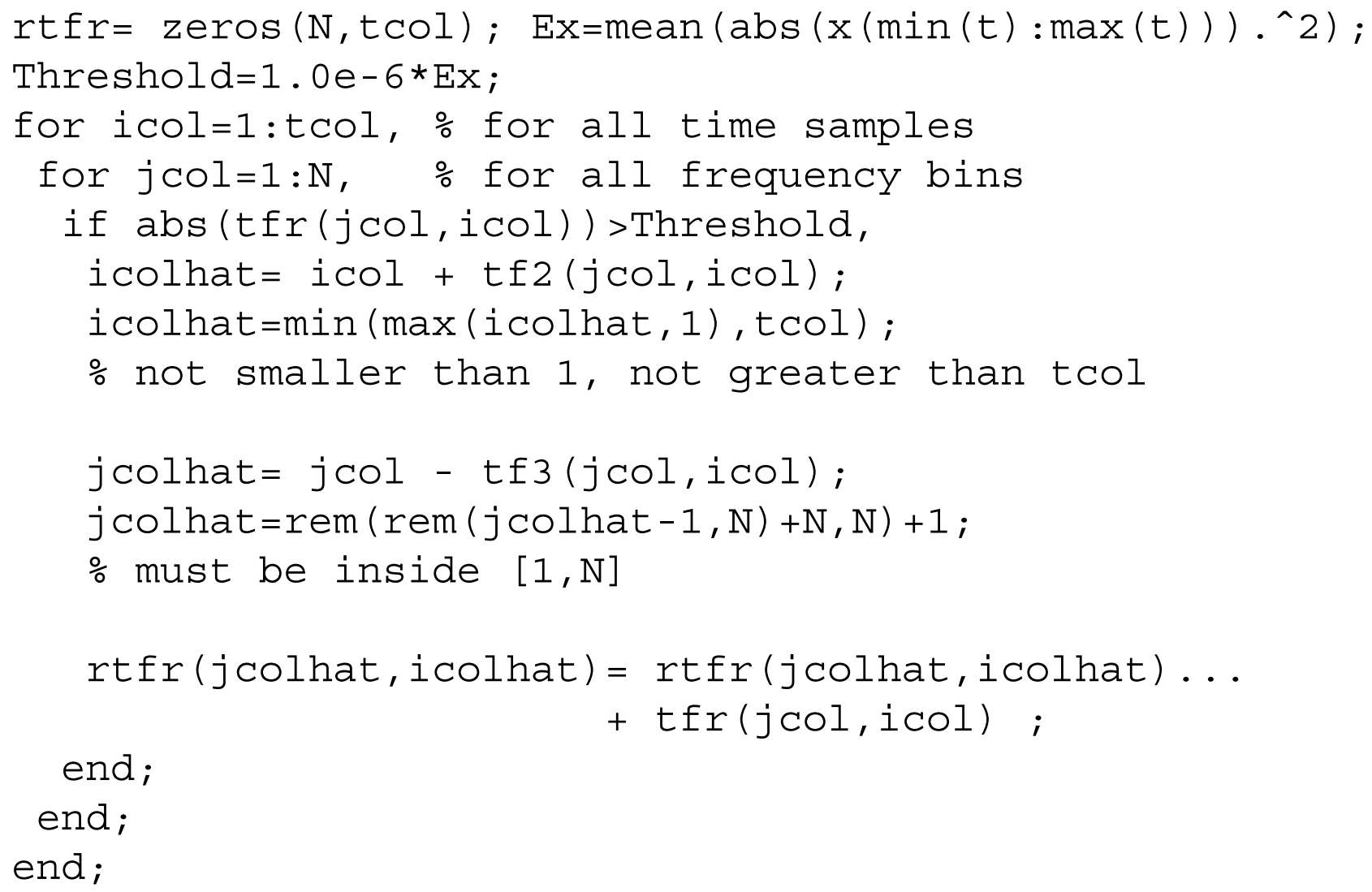

It should be emphasized that unlike the first step, which greatly benefits from the vectorization capabilities of MATLAB, this second step is a slower process under the MATLAB environment, because of the two embedded loops. Faster implementations written in the American National Standards Institute (ANSI) C language are also available at:

www-sigproc.eng.cam.ac.uk/md283/toolbox/Ctftbeng.html

\subsubsection{Computing window derivative}

The first step of this algorithm requires, however, an evaluation of the derivative of the analysis window function $h(t)$. An exact expression of this derivative could be obtained from the analytical expression of $h(t)$. This solution is too restrictive, because it does not allow someone to use self-made windows, whose derivatives may be difficult to get analytically. This is the reason why instead of the exact expression we use an accurate approximation, obtained by a centered first-order derivative. For this, $h(t)$ is modeled on its finite length support $[-T / 2, T / 2]$ as:

$$
h(t)= \begin{cases}\alpha+\beta t+h_{0}(t) & \text { for }|t| \leq T / 2 \\ 0 & \text { for }|t|>T / 2\end{cases}
$$

where $\alpha=(h(T / 2)+h(-T / 2)) / 2$ and $\beta=(h(T / 2)-h(-T / 2)) / T$ are, the offset and the slope of a linear trend, respectively and $h_{0}(t)$ is continuous and zero valued at 


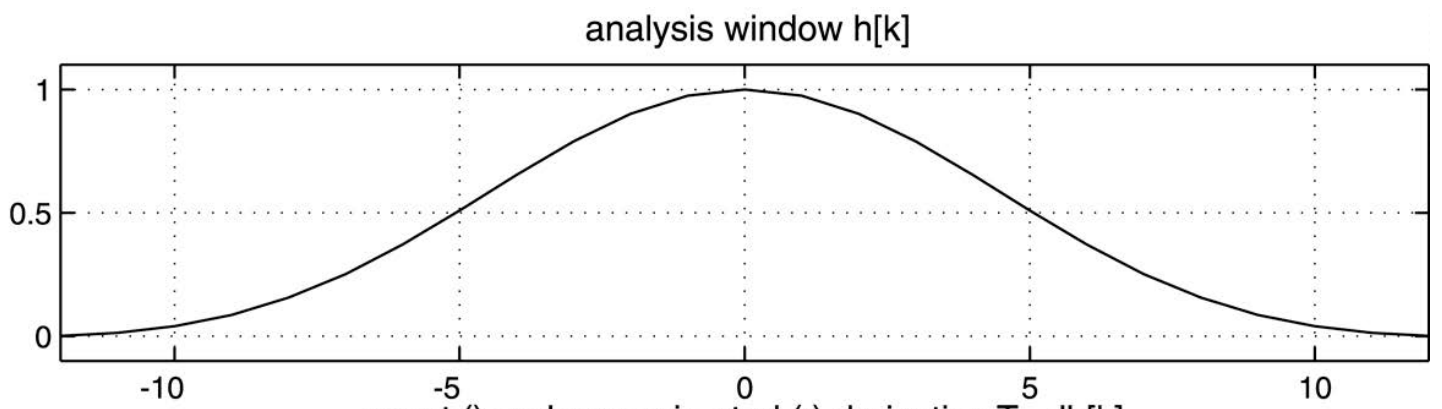

exact () and approximated (:) derivative $\mathrm{T}_{\mathrm{s}} \mathrm{dh}[\mathrm{k}]$
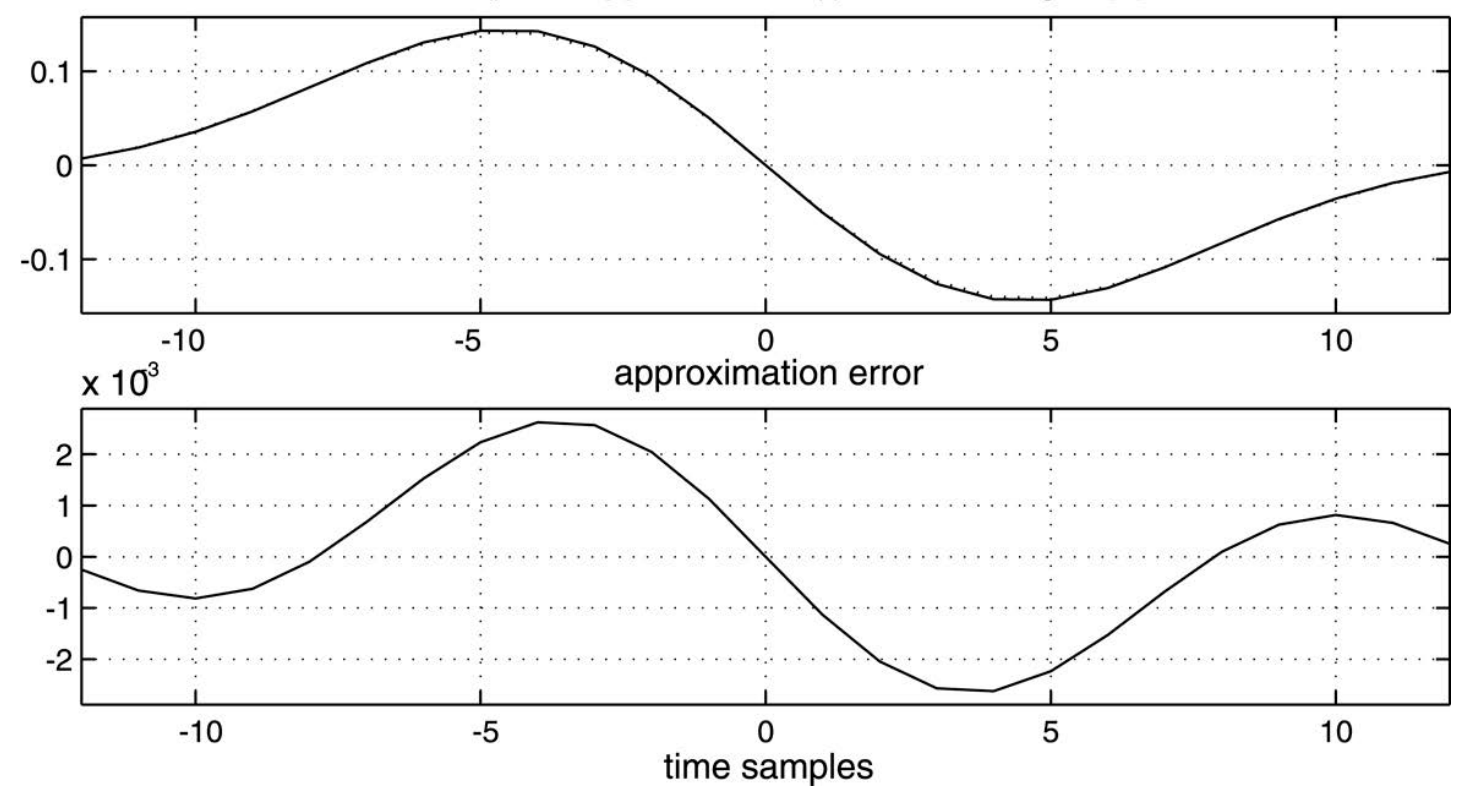

\section{FIGURE 5.7}

Window derivative approximation. For a Blackman window of 25 points, the difference between the exact and the approximated derivative is very weak.

the window edges $-T / 2$ and $T / 2$. Under these conditions, $\frac{d h_{0}}{d t}(t)$ can be accurately approximated by a first-order difference, and $\frac{d h}{d t}(t)$ can be deduced according to:

$$
\frac{d h}{d t}(t)= \begin{cases}\alpha \delta(t+T / 2)-\alpha \delta(t-T / 2)+\beta+\frac{d h_{0}}{d t}(t) & \text { for }|t| \leq T / 2 \\ 0 & \text { for }|t|>T / 2\end{cases}
$$

To evaluate the quality of this approximation, the function about_dh compares the exact derivative of a Blackman window [21] with its approximation derived from Equation (5.27). For $\mathbf{N h = 2 5}$, the results are presented in Figure 5.7, showing a maximum error of $3 \times 10^{-3}$, leading to a relative error of less than $2 \%$. This error still decreases when the window length increases.

The framework presented here is the background of the function dwindow, which essentially reduces to:

function $\mathrm{Dh}=$ dwindow (h) ;

$\div \mathrm{h}$ is a row vector

[hrow, hcol] =size (h); Lh= (hrow-1) /2 ; 
\% compute the discontinuities on the border step_height $=($ h $(1)+h($ hrow $)) / 2$;

ramp $=($ h (hrow $)-h(1)) /($ hrow -1$)$;

$\%$ add zeros at both sides

h2 $=\left[0 ;\right.$ h-step_height-ramp* $(-$ Lh $:$ Lh $\left.) . '{ }^{\prime} ; 0\right]$;

$\mathrm{Dh}=(\mathrm{h} 2(3:$ hrow +2$)-\mathrm{h} 2(1: \mathrm{hrow})) / 2+$ ramp; $\%$ first order difference

$\operatorname{Dh}(1)=\mathrm{Dh}(1) \quad+$ step_height; $\frac{\circ}{\circ}$ add discontinuities Dh $($ hrow $)=$ Dh (hrow) - step_height ;

This function allows the analysis window to be given by a vector of numerical values, instead of chosen among a set of predefined possibilities (Hamming, Hann, Kaiser-Bessel, ... [21]). Thanks to these functions tfrrsp and dwindow, the reassigned spectrogram of a signal can be easily obtained by a few instructions, such as in the ones included in the function $\mathbf{s p r s p p l o t}(\mathbf{x}, \mathbf{d t}, \mathbf{N b}, \mathbf{N h}, \mathbf{c})$ presented in Section 5.2.3.1:

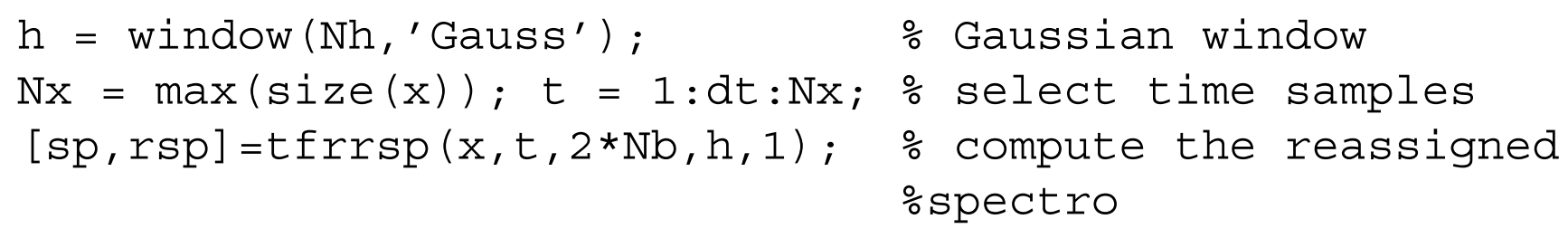

The sensitivity of the reassigned spectrogram to the choice of the window shape or length can therefore be easily studied by simply changing the parameters of the function window.

\subsubsection{Reassigning other time-frequency distributions}

\subsubsection{Reassigned smoothed pseudo-Wigner-Ville distributions}

As mentioned in Section 5.2.2.1, spectrograms suffer from a difficult trade-off between time and frequency resolutions, which make them irrelevant for several real case applications. Hopefully, other TF representations exist, which can also be reassigned. For instance, the smoothed pseudo-Wigner-Ville distribution (SPWV) [16] is a very flexible tool, which allows an independent adjustment of the time and frequency smoothings:

$$
\operatorname{SPWV}_{x}^{g, h}(t, \omega):=\iint_{-\infty}^{+\infty} g(s-t) H(\xi-\omega) W_{x}(s, \xi) \frac{d s d \xi}{2 \pi}
$$

where $g$ and $h$ are two even smoothing windows with $h(0)=G(0)=1$. In that case, expressions in Equations (5.7) and (5.8) become:

$$
\hat{t}_{x}(t, \omega)=t+\frac{\operatorname{SPWV}_{x}^{g, t h}(t, \omega)}{\operatorname{SPWV}_{x}^{g, h}(t, \omega)}
$$



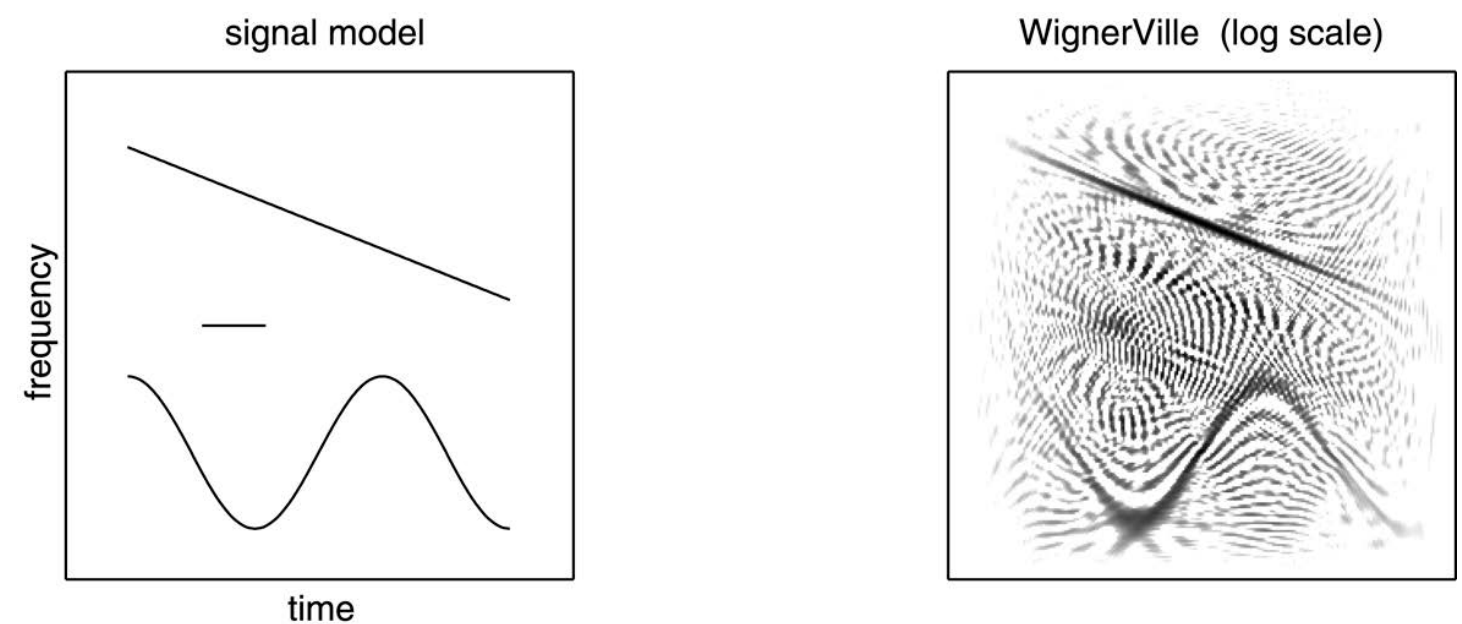

SPWV (log scale)

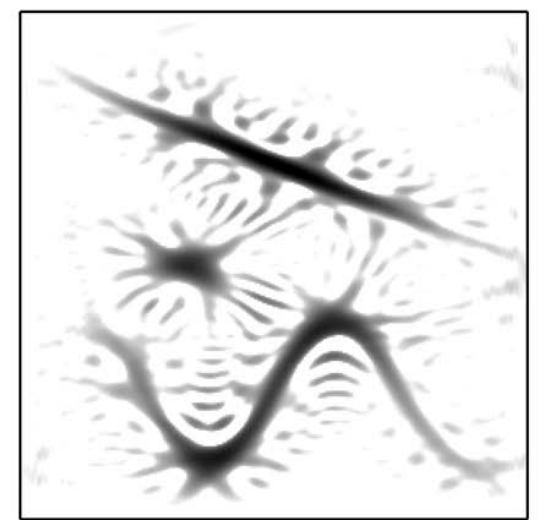

reassigned SPWV (log scale)

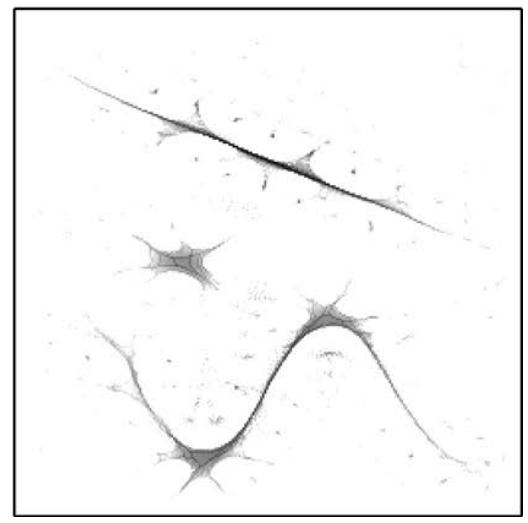

\section{FIGURE 5.8}

SPWV reassignment. This figure is similar to Figure 5.2, but the spectrogram and its reassigned version have been replaced by the SPWV and its reassigned version, computed for exactly the same signal realization. The improvement brought by this kind of representation is clearly evidenced.

$$
\hat{\omega}_{x}(t, \omega)=\omega-i \frac{\mathrm{SPWV}_{x}^{d g, h}(t, \omega)}{\operatorname{SPWV}_{x}^{g, h}(t, \omega)}
$$

These new expressions show that reassigning the SPWV distribution only requires two additional SPWV with particular smoothing windows. The resulting algorithm, presented in the Figure 2 of Reference [2] and implemented in the function tfrrspwv of the TF toolbox [3], has the same organization in two steps than the algorithm of the reassigned spectrogram:

- The first one computes the three SPWVs.

- The second one iteratively creates the reassigned smoothed pseudo-WignerVille distribution $\check{P}_{x}^{g, h}(t, \omega)$ by adding $P_{x}^{g, h}[n, m]$ to $\check{P}_{x}^{g, h}[\hat{n}, \hat{m}]$.

The relevance of this representation is supported by Figure 5.8, obtained by running model ITF 2; this new figure shows two new representations of exactly the same signal as the one used in Figure 5.2. The SPWV (bottom left) yields a better localization of the signal components than the spectrogram, and therefore its reassigned version 
(bottom right) is still closer to the idealized model and less sensitive to noise than the reassigned spectrogram. In this script, the SPWV and its reassigned version are simply obtained by the instructions:

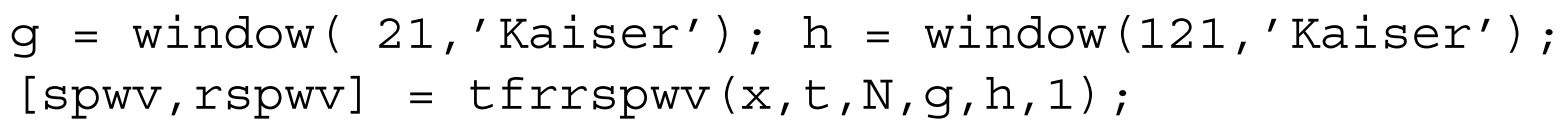

Improvements can easily be looked for by simply changing the definitions of $\mathbf{g}$ and $\mathbf{h}$.

\subsubsection{Reassigned scalograms}

The reassignment principle can also be applied to time-scale representations of the affine class [32]. A widely used member of this class is the scalogram, which is the squared modulus of the continuous wavelet transform:

$$
\begin{aligned}
\operatorname{SC}_{x}^{h}(t, a) & :=\left|\mathrm{CW}_{x}^{h}(t, a)\right|^{2} \\
\text { with } \quad \mathrm{CW}_{x}^{h}(t, a) & :=\frac{1}{\sqrt{|a|}} \int_{-\infty}^{+\infty} x(u) h^{*}\left(\frac{u-t}{a}\right) d u
\end{aligned}
$$

where $h(t)$ (called the mother wavelet) is the impulse response of a bandpass filter of central frequency $\omega_{0}$, and $a$ is a scale parameter, related to a frequency variable by the relationship $a=\omega_{0} / \omega$.

As the expression in Equation (5.6), the scalogram results from an affine smoothing of the WVD:

$$
\mathrm{SC}_{x}^{h}(t, a)=\iint_{-\infty}^{+\infty} W_{x}(s, \xi) W_{h}\left(\frac{s-t}{a}, a \xi\right) \frac{d s d \xi}{2 \pi}
$$

As evidenced by this expression, $\mathrm{SC}_{x}^{h}(t, a)$ can be interpreted as the summation of a whole set of energy measures $W_{x}(s, \xi)$ contained within a TF domain delimited by $W_{h}\left(\frac{s-t}{a}, a \xi\right)$. Instead of assigning this number to the geometric center of this domain, which does not depend on the analyzed signal, it seems more relevant to assign it to the center of gravity, defined by:

$$
\begin{aligned}
\hat{t}_{x}(t, a) & =\frac{1}{\mathrm{SC}_{x}^{h}(t, a)} \iint_{-\infty}^{+\infty} s W_{x}(s, \xi) W_{h}\left(\frac{s-t}{a}, a \xi\right) \frac{d s d \xi}{2 \pi} \\
\hat{\omega}_{x}(t, a):=\frac{\omega_{0}}{\hat{a}_{x}(t, a)} & =\frac{1}{\mathrm{SC}_{x}^{h}(t, a)} \iint_{-\infty}^{+\infty} \xi W_{x}(s, \xi) W_{h}\left(\frac{s-t}{a}, a \xi\right) \frac{d s d \xi}{2 \pi}
\end{aligned}
$$

The resulting reassigned scalogram, defined as:

$$
\check{\mathrm{SC}}_{x}^{h}\left(t^{\prime}, a^{\prime}\right):=\iint_{-\infty}^{+\infty} \mathrm{SC}_{x}^{h}(t, a) \delta\left(t-\hat{t}_{x}(t, a), a^{\prime}-\hat{a}_{x}(t, a)\right) \frac{a^{\prime 2} d t d a}{a^{2}}
$$

benefits both from the smoothing performed by the mother wavelet, and from the reassignment, which refocuses the scalogram on the squeezed signal description given by the WVD. 
From a computational point of view, the local centroids can efficiently be computed by means of two additional wavelet transforms, using two particular mother wavelets:

$$
\begin{array}{r}
\hat{t}_{x}(t, a)=t+\mathcal{R} e\left\{\frac{a \mathrm{CW}_{x}^{t h}(t, a)}{\mathrm{CW}_{x}^{h}(t, a)}\right\} \\
\hat{\omega}_{x}(t, a)=\frac{\omega_{0}}{\hat{a}_{x}(t, a)}=-\mathcal{I} m\left\{\frac{\mathrm{CW}_{x}^{d h}(t, a)}{\mathrm{CW}_{x}^{h}(t, a)}\right\}
\end{array}
$$

Several mother wavelet functions can be used. One of them is the Morlet wavelet:

$$
h(t)=\frac{1}{\sqrt{T}} e^{-\frac{t^{2}}{2 T^{2}}} e^{i \omega_{0} t}
$$

In that case, $d h(t)=\frac{t h(t)}{T^{2}}+i \omega_{0} h(t)$ and $\mathrm{CW}_{x}^{d h}(t, a)=-\frac{1}{T^{2}} \mathrm{CW}_{x}^{t h}(t, a)-i \omega_{0}$ $\mathrm{CW}_{x}^{h}(t, a)$. As for the Gabor spectrogram, only $\mathrm{CW}_{x}^{h}(t, a)$ and $a \mathrm{CW}_{x}^{t h}(t, a)$ need to be computed, because the expression in Equation (5.35) becomes:

$$
\hat{\omega}_{x}(t, a)=\frac{\omega_{0}}{\hat{a}_{x}(t, a)}=\frac{\omega_{0}}{a}+\frac{1}{a^{2} T^{2}} \mathcal{I} m\left\{\frac{a C W_{x}^{t h}(t, a)}{\mathrm{CW}_{x}^{h}(t, a)}\right\}
$$

Computer algorithms (as the one used in the function tfrrmsc) can therefore be deduced from the discrete-time versions of the following expressions:

$$
\begin{aligned}
\mathrm{CW}_{x}^{h}(t, a) & =\sqrt{\frac{|\omega|}{\omega_{0} T}} \int_{-\infty}^{+\infty} x(t+\tau) e^{-\frac{\omega^{2} \tau^{2}}{2 \omega_{0}^{2} T^{2}}} e^{-i \omega \tau} d \tau \\
a \mathrm{CW}_{x}^{t h}(t, a) & =\sqrt{\frac{|\omega|}{\omega_{0} T}} \int_{-\infty}^{+\infty} x(t+\tau) \tau e^{-\frac{\omega^{2} \tau^{2}}{2 \omega_{0}^{2} T^{2}}} e^{-i \omega \tau} d \tau
\end{aligned}
$$

with $a=\omega_{0} / \omega$. Because the Gaussian analyzing window used in these expressions depends on the frequency (or scale) parameter, FFT algorithms can no longer be used, resulting in much slower algorithms. It should be emphasized that $\omega_{0}$ and $T$ only appear through their product $\omega_{0} T$, which is the only degree of freedom of this representation. Increasing this parameter improves the frequency resolution and reduces the time resolution.

The representations obtained with the Morlet scalogram and its reassigned version for the signal taken as example through this chapter are presented on Figure 5.9. Because the sinusoidal FM requires a small time resolution at low frequencies, the linear FM component at higher frequencies has a very broad frequency localization. This illustrates the fact that a proper choice of a TF representation can only be deduced from a study of the signal structure. The reassigned version, however, remains very close to the signal model. 


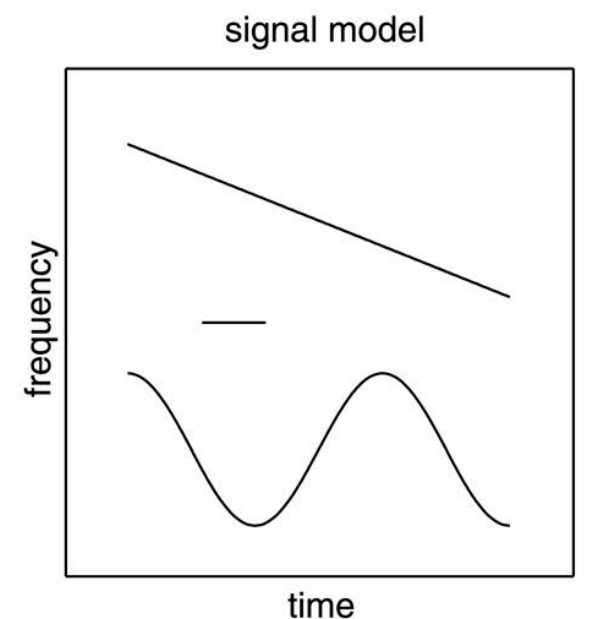

Morlet Scalogram (log scale), $f_{0} T=1.5$

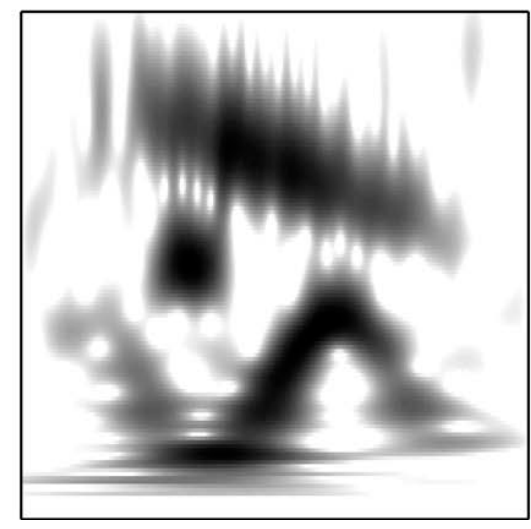

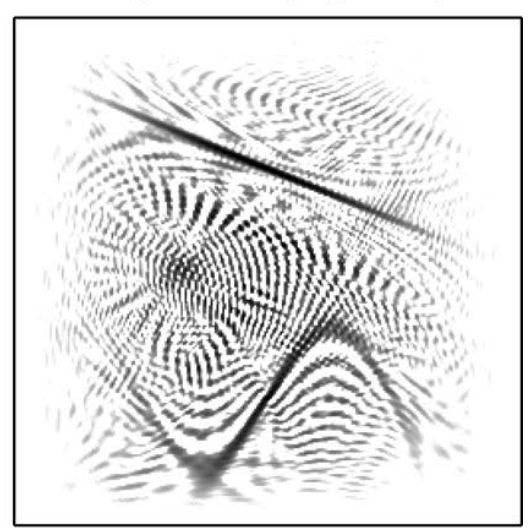

reassigned MSC (log scale)

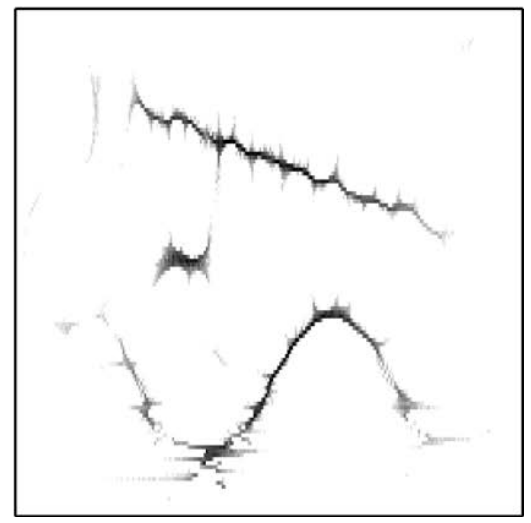

\section{FIGURE 5.9}

Scalogram reassignment. This figure is similar to Figure 5.2, but the spectrogram and its reassigned version have been replaced by the Morlet scalogram and its reassigned version, computed for exactly the same signal realization. The use of this kind of representation for the analyzed signal does not seem to be relevant.

\subsection{Real case studies}

The principles and the algorithms presented in Sections 5.2 and 5.3 can be used efficiently in real case situations. This section briefly presents two applications chosen among many possible ones.

The first one deals with nondestructive testing of metallic cables for suspension bridges. For this, a short wave is emitted by a magnetostrictive transducer, and propagated through a cable under test. The reflections on the normal and defective edges are then received by a sensor [1]. The resulting signal is shown on top of Figure 5.10, whereas reassigned spectrograms in the bottom part give a close look to the fifth and ninth reflected waves, showing precisely the dispersion of the wave through the metallic cable.

The second application deals with sensorless control of direct current (DC) motors. The electrical contacts and disconnections made by the brushes on the commutator create low-amplitude components in the stator current signal, with frequencies related 

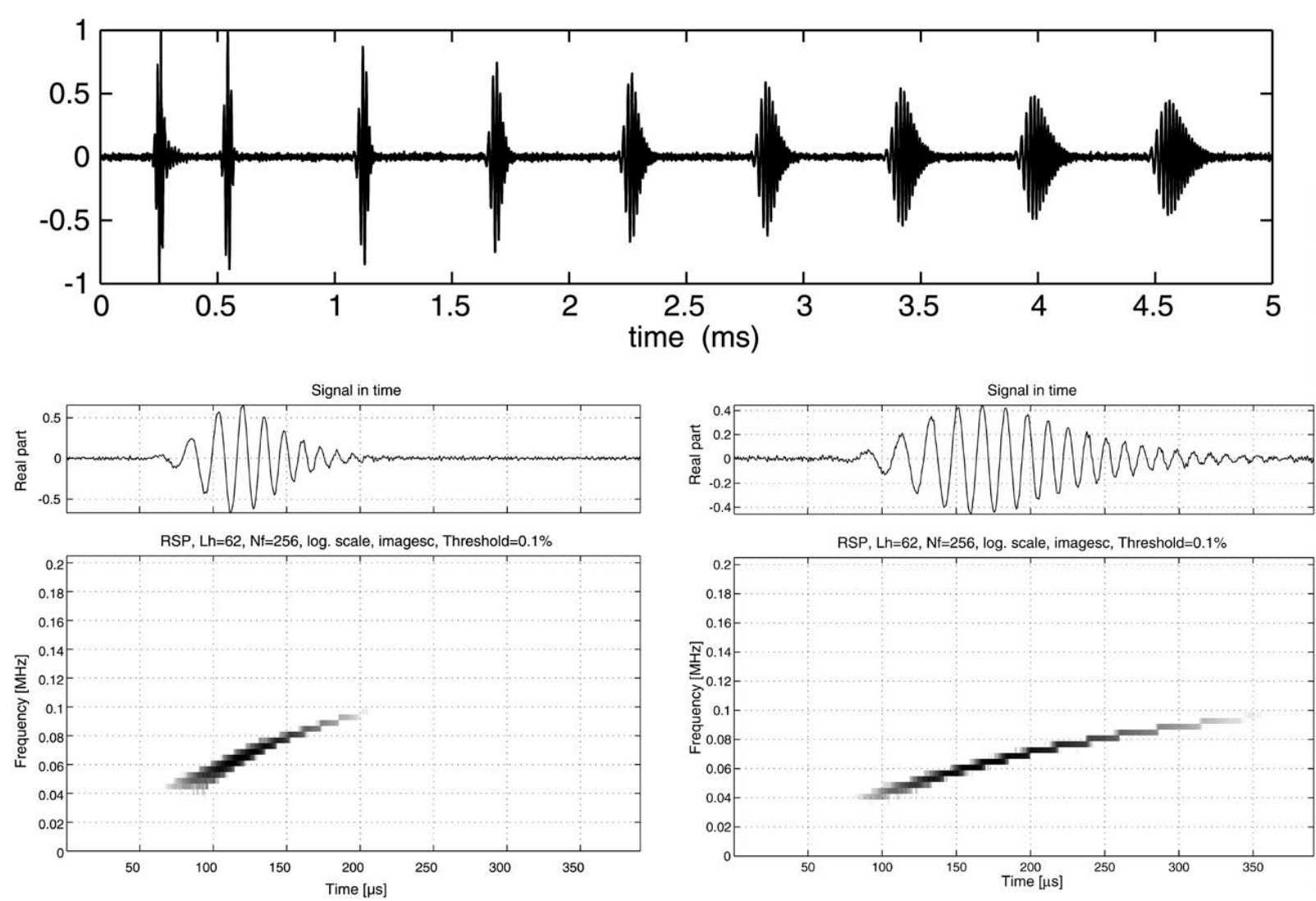

\section{FIGURE 5.10}

Mechanical waves propagating through a metallic cable. This figure shows the signal received by a sensor (top), and the TF analysis of the fifth (left) and ninth (right) reflections, from which an accurate study of the wave dispersion along the metallic cable can be done.

to the rotor speed [4]. The IFs of these components can be used to estimate the motor speed and to avoid the use of a mechanical sensor in a speed control loop. To illustrate the feasibility of this approach, Figure 5.11 shows the reassigned spectrogram of the stator current during a motor stopping. This representation clearly shows three signal components with almost linearly varying frequency. The higher the frequency is, the higher the chirping rate and therefore the better the estimation of the deceleration, but also the weaker the signal components.

Finally, a very interesting application in musical signal synthesis can also be found at:

\section{http://www.cerlsoundgroup.org/Loris}

\subsection{Conclusions}

Reassignment is a process having the goal of building readable TF representations, by first rubbing out oscillatory interferences, and then squeezing the remaining energy contributions to refocus them on the signal components. Reassignment is a very 

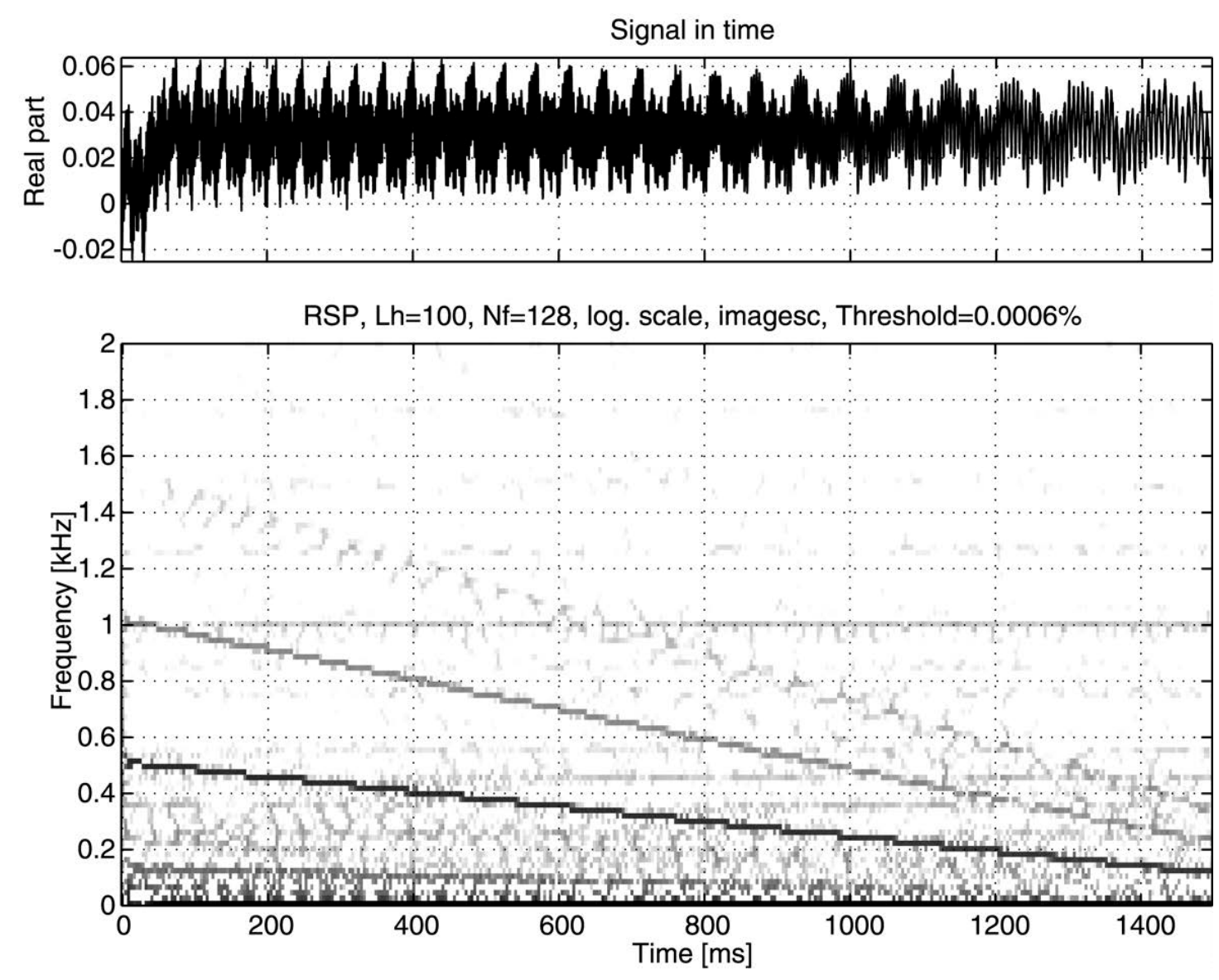

\section{FIGURE 5.11}

Rotor speed estimation from the stator current. This figure shows the TF analysis (reassigned spectrogram) of the current signal of a DC motor, evidencing AC components whose instantaneous frequencies are related to the rotor speed.

general principle with efficiency that has been rigorously proved, and that applies to a wide class of TF distributions, yielding an easy-to-read TF analysis, especially at high SNR. Efficient algorithms are available, thus allowing its practical use in effective signal analysis applications.

Beyond TF analysis, useful information on the signal structure can also be extracted from the reassignment operators, and used in a signal processing application. On the one hand, it can be applied to chirp detection, because we have shown [10] that reassignment can be used as the key element of an optimal detector. On the other hand, it can be used as the background of a TF partitioning algorithm for multicomponent signals $[8,11]$.

\section{References}

[1] J.-C. Aime, M. Brissaud, and L. Laguerre, Generation and Detection of Elastic Guided Waves with Magnetoelastic Device for the Nondestructive Evaluation of Steel Cables and Bars, in Proceedings 15th World Conference on Nondestructive Testing, Rome, Italy, 2000. 
[2] F. Auger and P. Flandrin, Improving the readability of time-frequency and time-scale representations by the reassignment method, IEEE Trans. Signal Process., SP-43(5), 1068-1089, 1995.

[3] F. Auger, P. Flandrin, P. Gonçalvès, and O. Lemoine, Time-frequency toolbox for MATLAB, user's guide and reference guide, available at http://iut-saintnazaire.univ-nantes.fr/ $\sim$ auger/tftb.html.

[4] F. Auger and M. Hilairet, Suivi de Raies Spectrales Avec un Faible Coût de Calcul (in French), in Proceedings 17ème Colloque GRETSI, Vannes, France, 1999, pp. 909-912.

[5] R. Carmona, H.L. Hwang, and B. Torrésani, Practical Time-Frequency Analysis, Academic Press, San Diego, CA, 1998.

[6] E. Chassande-Mottin, F. Auger, and P. Flandrin, Supervised Time-Frequency Reassignment, in Proceedings IEEE International Symposium on TimeFrequency and Time-Scale Analysis, Paris, France, 1996, pp. 517-520.

[7] E. Chassande-Mottin, I. Daubechies, F. Auger, and P. Flandrin, Differential reassignment, IEEE Signal Process. Lett., SPL-4(10), 293-294, 1997.

[8] E. Chassande-Mottin, F. Auger, I. Daubechies, and P. Flandrin, Partition du Plan Temps-Fréquence et Réallocation (in French), in Proceedings 16ème Colloque GRETSI, Grenoble, France, 1997, pp. 1447-1450.

[9] E. Chassande-Mottin, F. Auger, and P. Flandrin, On the statistics of spectrogram reassignment, Multidim. Syst. Signal Process., 9(4), 355-362, 1998.

[10] E. Chassande-Mottin and P. Flandrin, On the time-frequency detection of chirps, Appl. Comp. Harm. Anal., 6(9), 252-281, 1999.

[11] E. Chassande-Mottin, F. Auger, and P. Flandrin, Time-frequency/time-scale reassignment, in Wavelets and Signal Processing, L. Debnath, Ed., Birkhäuser, Boston, to appear 2001.

[12] L. Cohen, Time-Frequency Analysis, Prentice Hall, Englewoods Cliffs, NJ, 1995.

[13] N. Delprat, B. Escudié, P. Guillemain, R. Kronland-Martinet, P. Tchamitchian, and B. Torrésani, Asymptotic wavelet and Gabor analysis: extraction of instantaneous frequencies, IEEE Trans. Inf. Theory, IT-38(2), 644-673, 1992.

[14] I. Djurovic and L.J. Stankovic, Time-frequency representation based on the reassigned S-method, Signal Process., 77(1), 115-120, 1999.

[15] K. Fitz, L. Haken, and P. Christensen, Transient preservation under Transformation in an Additive Sound Model, in Proceedings International Computer Music Conference, Berlin (D), 2000, pp. 392-395.

[16] P. Flandrin, Time-Frequency/Time-Scale Analysis, Academic Press, San Diego, CA, 1999. 
[17] D. Friedman, Instantaneous Frequency Distribution vs. Time : An Interpretation of the Phase Structure of Speech, in Proceedings of the IEEE International Conference on Acoustics, Speech, and Signal Processing, Tampa, FL, 1985, 1121-1124.

[18] V. Gibiat, F. Wu, P. Perio, and S. Chantreuil, Analyse spectrale différentielle (A.S.D.), C. R. Acad. Sci. Paris, sér. II, 294, 633-636, 1982.

[19] P. Guillemain and R. Kronland-Martinet, Horizontal and Vertical Ridges associated to Continuous Wavelet Transforms, in Proceedings of the IEEE International Symposium on Time-Frequency and Time-Scale Analysis, Victoria, Canada, 1992, pp. 63-66.

[20] P. Guillemain, and R. Kronland-Martinet, Characterization of acoustic signals through continuous linear time-frequency representations, Proc. IEEE, 84(4), 561-587, 1996.

[21] F.J. Harris, On the use of windows for harmonic analysis with the discrete Fourier transform, Proc. IEEE, 66(1), 51-83, 1978.

[22] F. Hlawatsch and G.F. Boudreaux-Bartels, Linear and quadratic timefrequency signal representations, IEEE Signal Process. Mag., 21-67, 1992.

[23] F. Hlawatsch and P. Flandrin, The interference structure of Wigner distributions and related time-frequency signal representations, in The Wigner Distribution: Theory and Applications in Signal Processing, W. Mecklenbräuker and F. Hlawatsch, Eds., Elsevier, Amsterdam, 1998, pp. 59-133.

[24] J.-M. Innocent and B. Torrésani, Wavelets and binary coalescences detection, Appl. Comp. Harm. Anal., 4(2), 113-116, 1997.

[25] K. Kodera, C. De Villedary, and R. Gendrin, A new method for the numerical analysis of nonstationary signals, Phys. Earth Plan. Int., 12, 142-150, 1976.

[26] K. Kodera, Analyse Numérique de Signaux Géophysiques Non-Stationnaires, Thèse de doctorat, Université de Paris VI, France, 1976.

[27] K. Kodera, R. Gendrin, and C. De Villedary, Analysis of time-varying signals with small BT values, IEEE Trans. Acoust. Speech Signal Process., ASSP26(1), 64-76, 1978.

[28] R.J. McAulay and T.F. Quatieri, Speech analysis-synthesis based on a sinusoidal representation, IEEE Trans. Acoust. Speech Signal Process., ASSP34(4), 744-754, 1986.

[29] S. Maes, The Synchrosqueezed Representation Yields a New Reading of the Wavelet Transform, in Proceedings SPIE 95 on OE/Aerospace Sensing and Dual Use Photonics, Orlando, FL, 1995, pp. 532-559.

[30] S. Mallat, A Wavelet Tour of Signal Processing, Academic Press, New York, 1998. 
[31] C. Richard and R. Lengellé, Joint recursive implementation of time-frequency representations and their modified version by the reassignment method, Signal Process., 60(2), 163-179, 1997.

[32] O. Rioul and P. Flandrin, Time-scale energy distributions: a general class extending wavelet transforms, IEEE Trans. Signal Process., SP-40(7), 1746-1757, 1992.

[33] J.-M. Tribolet, A new phase unwrapping algorithm, IEEE Trans. Acoust. Speech Signal Process., ASSP-25(2), 170-177, 1977. 